\title{
MHD Free Convection Effects of Radiating Memory Flow
}

\author{
Syed Amanullah Hussaini ${ }^{1}$ M.V Ramana Murthy ${ }^{2}$ and Rafiuddin ${ }^{3}$ \\ ${ }^{1} \mathrm{M}$ J College of Engineering \& Technology, Department of Mathematics, Hyderabad, India \\ Email: amanullahsd@gmail.com \\ ${ }^{2}$ Osmania University, Department of Mathematics, Hyderabad, India \\ Email: mv_rm@rediffmail.com \\ ${ }^{3}$ CVR College of Engineering, Department of H \& S, Ibrahimpatam (M ), R.R.District, A.P., India \\ Email: rafiuddin2008@gmail.com
}

\begin{abstract}
Two dimensional unsteady radiating oscillatory memory flow past an infinite plane porous wall is investigated. Expressions for the mean velocity and the mean temperature are obtained. The effects of various parameters such as Permeability parameter $(\mathbf{K})$, Grashoff number $\left(G_{r}\right)$, Hartmann number $(M)$, Radiation parameter $(\mathbf{N})$, Prandle number $\left(\mathbf{P}_{\mathbf{r}}\right)$ are depicted graphically. Nusselt number $(\mathrm{Nu})$ and Skin friction $\left(\mathrm{C}_{\mathrm{f}}\right)$ are discussed by tables.
\end{abstract}

Index Terms - Suction, Porous medium, Walter's liquid- B and Rosseland's approximation.

\section{INTRODUCTION}

The study of flow of electrically conducting fluid, the so-called magneto-hydrodynamics (MHD) has a lot of attention due to its diverse applications in astrophysics and geophysics. It is important to study the effect of the interaction of magnetic field on the temperature distribution and heat transfer when the fluid is not only electrically conducting but also when it is capable of emitting and absorbing thermal radiation. Heat transfer by thermal radiation is important when we are concerned with space technology applications and in power engineering. Seddeek [3] studied the effects of radiation and variable viscosity on a MHD free convection flow past an semi-infinite flat plate with an aligned magnetic field in the case of unsteady flow. Mazumdar and Deka [4] investigated MHD flow past an impulsively started infinite vertical plate in presence of thermal radiation. Analytical closed-form solution of the unsteady hydro-magnetic natural convection heat and mass transfer flow of a rotating, incompressible, viscous Boussinesq fluid was presented by Mbeledogu and Ogulu [5]. Mbeledogu et al. [6] also studied the free convection flow of a compressible Boussinesq fluid under the simultaneous action of buoyancy and transverse magnetic field. Mohamed and Abo- Dahab [7] analysis, the effects of chemical reaction and thermal radiation on hydromagnetic free convection heat and mass transfer for a micropolar fluid via a porous medium bounded by a semi-infinite vertical porous plate in the presence of heat generation. Natural convection of an electrically conducting and radiating fluid in the presence of an external magnetic field is investigated numerically by Young [8]. Zueco and Anwar [9] studied the steady-state, magneto hydrodynamic, optically thick, dissipative gas boundary layer flow and heat transfer past a non-isothermal porous wedge embedded in a scattering, homogenous, isotropic Darcy_Forchheimer porous medium, with significant thermal radiation effects in the presence of heat sink/sources and surface transpiration, in a $(\mathrm{x}, \mathrm{y})$ coordinate system. Ahmed and Sarmah [10] analyzed the joint effect of thermal radiation and transverse magnetic field on an unsteady convection and mass transfer flow of an incompressible viscous electrically conducting fluid past a suddenly fixed vertical plate. Ishak [11] worked mixed convection boundary layer flow over a horizontal plate with thermal radiation. Soret and Dufour effects on free convection flow of non-Newtonian fluids along a vertical plate embedded in a porous medium with thermal radiation were studied by Tai and Char [12]. Israel - Cookey and Nwaigwe [13] considered unsteady MHD flow of a radiating fluid over a vertical moving heated porous plate with time dependent suction. Narahari [14] studied the effects of thermal radiation and free convection currents on the unsteady Couette flow between two vertical parallel plates with constant heat flux at one boundary. Israel Cookey et al. [15] investigated the combined effects of radiative heat transfer and a transverse magnetic field on steady flow of an electrically conducting optically thin fluid through a horizontal channel filled with porous medium and non- uniform temperatures at the walls. Singh et al. [16] studied the effect of surface mass transfer on MHD mixed convection flow past a heated vertical flat permeable surface in the presence of thermophoresis, radiative heat flux and heat source/sink. Ahmed [17] given the exact solution to the problem of MHD transient free convection and mass 
transfer flow of a viscous, incompressible, and electrically conducting fluid past a suddenly started infinite vertical plate taking into account the thermal diffusion as well as the thermal radiation was presented. The heat and mass transfer characteristics of the unsteady electrically conducting fluid flow past a suddenly started vertical infinite flat plate studied by Turkyilmazoglu and Pop [18]. Free convective heat and mass transfer flow over a moving permeable flat vertical stretching sheet in the presence of non-uniform magnetic field is numerically investigated by Ferdows et al. [19].

Now we study the free convection effects on the fluctuating flow of radiating memory flow past an infinite porous wall with constant suction through porous medium.

\section{FORMULATION OF PROBLEM}

We consider the free convective flow due to fluctuating mean stream of memory fluid (Walter's Liquid Model-B) flow past an infinite plane, porous wall with constant suction at the surface. Let $y=0$ be the wall in the presence of magnetic field normal to the direction of flow. Let $\mathrm{u}$ and $\mathrm{v}$ be the velocity components along and normal to the wall.

We make the following assumptions:

1) That all the fluid properties are constant except the density in the buoyancy force term;

2) That the term due to the electrically dissipation is neglected in energy equation (2.2) as the magnetic field is not strong enough to cause Joule heating (electrical dissipation)

3) That the Eckert number $E_{c}$ and the magnetic Renold's numbers are small so that the induced magnetic field can be neglected. The $\mathrm{x}$-axis is taken along the surface in the upwards direction and y-axis is taken normal to it.

As the bounding surface is infinite in length, all the variables are functions of $y$ alone. By the usual boundary layer approximation the basic equation for unsteady flow are

$$
\begin{aligned}
& \mathrm{v}=-\mathrm{v}_{0} \\
& \begin{aligned}
& \frac{\partial \mathrm{u}}{\partial \mathrm{t}}-\mathrm{v}_{0} \frac{\partial \mathrm{u}}{\partial \mathrm{y}}=\frac{\mathrm{dU}}{\mathrm{dt}}+\mathrm{g} \beta_{1}\left(\mathrm{~T}-\mathrm{T}_{\infty}\right)+\vartheta \frac{\partial^{2} \mathrm{u}}{\partial \mathrm{y}^{2}} \\
&-\frac{\beta}{\rho}\left[\frac{\partial^{3} \mathrm{u}}{\partial \mathrm{t} \partial \mathrm{y}^{2}}-\mathrm{v}_{0} \frac{\partial^{3} \mathrm{u}}{\partial \mathrm{y}^{3}}\right]-\frac{\vartheta \mathrm{u}}{\mathrm{K}} \\
&-\left(\frac{\sigma \mu_{\mathrm{e}}^{2} \mathrm{H}_{0}^{2}}{\rho}\right) \mathrm{u}
\end{aligned}
\end{aligned}
$$

$$
\begin{gathered}
\frac{\partial \mathrm{T}}{\partial \mathrm{t}}-\mathrm{v}_{0} \frac{\partial \mathrm{T}}{\partial \mathrm{y}}=\frac{\kappa}{\rho \mathrm{C}_{\mathrm{p}}} \frac{\partial^{2} \mathrm{~T}}{\partial \mathrm{y}^{2}}+\frac{\vartheta}{\mathrm{C}_{\mathrm{p}}}\left(\frac{\partial \mathrm{u}}{\partial \mathrm{y}}\right)^{2} \\
-\frac{1}{\rho \mathrm{C}_{\mathrm{p}}}\left(\frac{\partial \mathrm{q}_{\mathrm{r}}}{\partial \mathrm{y}}\right)
\end{gathered}
$$

where the Rosseland's approximation (Brewster[2]) is used, which leads to

$$
\mathrm{q}_{\mathrm{r}}=\frac{-4 \sigma^{*}}{3 \mathrm{k}^{*}} \frac{\partial \mathrm{T}^{4}}{\partial \mathrm{y}}
$$

The boundary conditions are:

$$
\begin{aligned}
& u(0, t)=0, T(0, t)=T_{w}, T(\infty, t)=T_{\infty} \\
& u(y, t)=U=U_{0}\left(1+\varepsilon e^{i \omega t}\right) \text { as } y \rightarrow \infty
\end{aligned}
$$

We assumed that the temperature differences within the flow are sufficiently small such that $\mathrm{T}^{4}$ may be expressed as a linear function of the temperature. This was accomplished by expanding $\mathrm{T}^{4}$ in a Taylor series about $\mathrm{T}_{\infty}$ and neglecting higher-order terms. Thus,

$$
\mathrm{T}^{4} \cong 4 \mathrm{~T}_{\infty}^{3} \mathrm{~T}-3 \mathrm{~T}_{\infty}^{4}
$$

Using (2.4) and (2.6) in (2.3) gives

$$
\begin{aligned}
\frac{\partial \mathrm{T}}{\partial \mathrm{t}}-\mathrm{v}_{0} \frac{\partial \mathrm{T}}{\partial \mathrm{y}}= & \frac{\kappa}{\rho \mathrm{C}_{\mathrm{p}}} \frac{\partial^{2} \mathrm{~T}}{\partial \mathrm{y}^{2}}+\frac{\vartheta}{\mathrm{C}_{\mathrm{p}}}\left(\frac{\partial \mathrm{u}}{\partial \mathrm{y}}\right)^{2} \\
& +\frac{1}{\rho \mathrm{C}_{\mathrm{p}}}\left(\frac{16 \sigma^{*} \mathrm{~T}_{\infty}^{3}}{3 \mathrm{kk}^{*}}\right)
\end{aligned}
$$

Introducing the following non-dimensional quantities:

$$
\begin{gathered}
\bar{y}=\frac{\mathrm{yv}_{0}}{\vartheta}, \overline{\mathrm{t}}=\frac{\mathrm{tv} \mathrm{v}_{0}^{2}}{\vartheta}, \overline{\mathrm{K}}=\frac{\mathrm{Kv} \mathrm{v}_{0}^{2}}{\vartheta^{2}}, \bar{\omega}=\frac{\vartheta \omega}{\mathrm{v}_{0}^{2}} \\
\mathrm{M}=\frac{\sigma \vartheta \mathrm{H}_{0}^{2} \mu_{\mathrm{e}}^{2}}{\rho \mathrm{v}_{0}^{2}}, \quad \mathrm{~N}=\frac{16 \sigma^{*} \mathrm{~T}_{\infty}^{3}}{3 \mathrm{k} \mathrm{k}^{*}}, \overline{\mathrm{u}}=\frac{\mathrm{u}}{\mathrm{U}_{0}^{\prime}} \\
\mathrm{W}=\frac{\mathrm{u}(\mathrm{t})}{\mathrm{U}_{0}}, \quad \mathrm{G}_{\mathrm{r}}=\frac{\mathrm{g} \beta_{1}\left(\mathrm{~T}_{\mathrm{w}}-\mathrm{T}_{\infty}\right)}{\mathrm{U}_{0} \mathrm{v}_{0}^{4}}, \\
\mathrm{R}_{\mathrm{m}}=\frac{\beta \mathrm{v}_{0}^{2}}{\vartheta^{2}}, \quad \mathrm{E}=\frac{\mathrm{u}_{0}^{2}}{\mathrm{C}_{\mathrm{p}}\left(\mathrm{T}_{\mathrm{w}}-\mathrm{T}_{\infty}\right)} \\
\mathrm{P}_{\mathrm{r}}=\frac{\vartheta \rho \mathrm{C}_{\mathrm{p}}}{\mathrm{K}}, \quad \theta=\frac{\mathrm{T}-\mathrm{T}_{\infty}}{\mathrm{T}_{\mathrm{w}}-\mathrm{T}_{\infty}}
\end{gathered}
$$


Using equation (2.2) into (2.3), then with the help of (2.8), the following equations are obtained

$$
\begin{gathered}
\frac{\partial \mathrm{u}}{\partial \mathrm{t}}-\frac{\partial \mathrm{u}}{\partial \mathrm{y}}=\frac{\mathrm{dW}}{\mathrm{dt}}+\mathrm{G}_{\mathrm{r}} \theta+\frac{\partial^{2} \mathrm{u}}{\partial \mathrm{y}^{2}}-\mathrm{R}_{\mathrm{m}}\left(\frac{\partial^{3} \mathrm{u}}{\partial \mathrm{t} \partial \mathrm{y}^{2}}-\frac{\partial^{3} \mathrm{u}}{\partial \mathrm{y}^{3}}\right) \\
-\mathrm{u}\left(\frac{1}{\mathrm{~K}}+\mathrm{M}\right) \\
\frac{\partial \theta}{\partial \mathrm{t}}-\mathrm{E}\left(\frac{\partial \mathrm{u}}{\partial \mathrm{y}}\right)^{2}=\left(\frac{1+\mathrm{N}}{\mathrm{P}_{\mathrm{r}}}\right) \frac{\partial^{2} \theta}{\partial \mathrm{y}^{2}}+\frac{\partial \theta}{\partial \mathrm{y}}
\end{gathered}
$$

The corresponding boundary conditions becomes

$$
\begin{array}{r}
\theta=1, \quad \mathrm{u}=0 \quad \text { at } \quad \mathrm{y}=0 \\
\theta \rightarrow 0, \quad \mathrm{w} \rightarrow \mathrm{W}(\mathrm{t}) \text { as } \mathrm{y} \rightarrow \infty
\end{array}
$$

Free-stream boundary condition

$$
\mathrm{W}(\mathrm{t})=\left(1+\varepsilon \mathrm{e}^{\mathrm{i} \omega \mathrm{t}}\right) \quad \text { as } \mathrm{y} \rightarrow \infty
$$

Where

$\rho \quad$ Density of fluid,

$\beta \quad$ Kinematic visco-elasticity,

$\beta_{1} \quad$ Coefficient of volume expansion,

$\mathrm{H}_{\mathrm{o}}$ Magnetic intensity,

$\mu_{e} \quad$ Magnetic permeability,

$\mathrm{R}_{\mathrm{m}}$ Magnetic Renolds number,

$\vartheta$ Kinematic viscosity,

K Permeability parameter,

$\mathrm{C}_{\mathrm{p}}$ Specific heat at constant pressure,

$\mathrm{N}$ Thermal radiation parameter,

$\mathrm{K}$ Thermal conductivity,

$G_{r}$ Grashoff number,

$\mathrm{P}_{\mathrm{r}}$ Prandtl number,

M Hartmann number,

E Eckert number,

q Heat flux,

$\sigma$ Electric conductivity,

$\sigma^{*} \quad$ Stephan-Boltzman constant,

$\mathrm{k}^{*}$ Mean absorption coefficient,

$\mathrm{f}_{1}=\left(\frac{\mathrm{Pr}_{\mathrm{r}}}{1+\mathrm{N}}\right)$

\section{SOLUTION OF THE PROBLEM}

Following Lighthill, [1] we put velocity and temperature field as

$$
\begin{gathered}
u=u_{0}+\varepsilon e^{i \omega t} u_{1} \\
\theta=\theta_{0}+\varepsilon e^{i \omega t} \theta_{1}
\end{gathered}
$$

$$
W(t)=\left(1+\varepsilon e^{i \omega t}\right)
$$

Where ' $\omega$ ' is the frequency and ' $\varepsilon$ ' is very small quantity $<<1$.

Now making use of (3.1) and (3.2) into the equations (2.9) and (2.10), equating harmonic and non-harmonic terms, we get

Zeroth order

$$
\begin{aligned}
& \mathrm{R}_{\mathrm{m}} \mathrm{u}_{0}^{\prime \prime \prime}+\mathrm{u}_{0}^{\prime \prime}+\mathrm{u}_{0}^{\prime}=-\mathrm{M}_{1} \mathrm{u}_{0}=-\mathrm{G}_{\mathrm{r}} \theta_{0} \\
& \left(\frac{1+\mathrm{N}}{\mathrm{P}_{\mathrm{r}}}\right) \theta_{0}^{\prime \prime}+\theta_{0}^{\prime}=-\mathrm{E}\left(\mathrm{u}_{0}^{\prime}\right)^{2}
\end{aligned}
$$

First order

$$
\begin{gathered}
\mathrm{R}_{\mathrm{m}} \mathrm{u}_{1}^{\prime \prime \prime}+(1-\operatorname{si\omega }) \mathrm{u}_{1}^{\prime \prime}+\mathrm{u}_{1}^{\prime}-\left(\mathrm{M}_{1}+\mathrm{i} \omega\right) \mathrm{u}_{1} \\
=-\mathrm{i} \omega-\mathrm{u}_{0}^{\prime}-\mathrm{G}_{\mathrm{r}} \theta_{1} \\
\theta_{1}^{\prime \prime}+\mathrm{f}_{1} \theta_{1}^{\prime}-\mathrm{f}_{1} \mathrm{i} \omega \theta_{1}=-\mathrm{f}_{1}\left[\theta_{0}+2 E \mathrm{Eu}_{0}^{\prime} \mathrm{u}_{1}^{\prime}\right]
\end{gathered}
$$

Boundary conditions are

$$
\begin{aligned}
& \theta_{0}=1, \theta_{1}=\mathrm{u}_{0}=\mathrm{u}_{1}=0 \text { as } \mathrm{y}=0 \\
& \theta_{0}=\theta_{1}=0, \mathrm{u}_{0}=\mathrm{u}_{1}=1 \text { as } \mathrm{y} \rightarrow \infty
\end{aligned}
$$

In equations (3.4) and (3.6), because of the presence of elasticity term, we get third order differential equation. To solve this, we need three boundary conditions but we have two. So following Beard and Walters (1964), we assume the solution as

$$
\begin{aligned}
& \mathrm{F}=\mathrm{F}_{1}(\mathrm{y})+\mathrm{R}_{\mathrm{m}} \mathrm{F}_{2}(\mathrm{y})+\mathrm{o}\left(R_{m}^{2}\right) \\
& \mathrm{u}_{0}(\mathrm{y})=\mathrm{u}_{01}(\mathrm{y})+\mathrm{R}_{\mathrm{m}} \mathrm{u}_{02}(\mathrm{y})+\mathrm{o}\left(R_{m}^{2}\right) \\
& \theta_{0}(\mathrm{y})=\theta_{01}(\mathrm{y})+\mathrm{R}_{\mathrm{m}} \theta_{02}(\mathrm{y})+\mathrm{o}\left(R_{m}^{2}\right) \\
& \mathrm{u}_{1}(\mathrm{y})=\mathrm{u}_{11}(\mathrm{y})+\mathrm{R}_{\mathrm{m}} \mathrm{u}_{12}(\mathrm{y})+\mathrm{o}\left(R_{m}^{2}\right) \\
& \theta_{1}(\mathrm{y})=\theta_{11}(\mathrm{y})+\mathrm{R}_{\mathrm{m}} \theta_{12}(\mathrm{y})+\mathrm{o}\left(R_{m}^{2}\right)
\end{aligned}
$$

Where $\mathrm{u}_{0}$ and $\theta_{0}$ are the mean velocity and mean temperature and $\mathrm{u}_{1}$ and $\theta_{1}$ are the fluctuating parts of the velocity and temperature respectively.

Using equations (3.10) into equations (3.4) - (3.7) equating the coefficients of different powers of $R_{m}$ and neglecting those of $R_{m}^{2}$

Thereafter considering differential equations related to mean parts 


$$
\begin{aligned}
& u_{01}^{\prime \prime}+u_{01}^{\prime}-M_{1} u_{01}=-G_{r} \theta_{01} \\
& u_{01}^{\prime \prime \prime}+u_{02}^{\prime \prime}+u_{02}^{\prime}-M_{1} u_{02}=-G_{r} \theta_{02} \\
& \theta_{01}^{\prime \prime}+f_{1} \theta_{01}^{\prime}=E_{1}\left(u_{01}^{\prime}\right)^{2} \\
& \theta_{02}^{\prime \prime}+f_{1} \theta_{02}^{\prime}=E_{1} 2 u_{01}^{\prime} u_{02}^{\prime}
\end{aligned}
$$

Boundary conditions are

$$
\begin{gathered}
\theta_{01}=1, \theta_{02}==\mathrm{u}_{01}=\mathrm{u}_{02} \rightarrow 0 \text { as } \mathrm{y} \rightarrow 0 \\
\theta_{01}=\theta_{02}=0, \mathrm{u}_{01}=1, \mathrm{u}_{02}=0 \text { as } \mathrm{y} \rightarrow \infty
\end{gathered}
$$

We solve the equations (3.11) - (3.14), we expand $\mathrm{u}$ and $\theta$ in powers of $\mathrm{E}$ because of Eckert number $\mathrm{E}$ is very small $(<<1)$ for incompressible fluid

$$
\mathrm{F}=\mathrm{F}_{1}+\mathrm{EF}_{2}
$$

Replacing $\mathrm{F}$ by $\mathrm{u}_{01}, \mathrm{u}_{02}, \theta_{01}, \theta_{02}$, we get

$$
\begin{gathered}
\mathrm{u}_{011}^{\prime \prime}+\mathrm{u}_{011}^{\prime}-\mathrm{M}_{1} \mathrm{u}_{011}=-\mathrm{G}_{\mathrm{r}} \theta_{011} \\
\mathrm{u}_{011}^{\prime \prime \prime}+\mathrm{u}_{021}^{\prime \prime}+\mathrm{u}_{021}^{\prime}=-\mathrm{M}_{1} \mathrm{u}_{021} \\
\mathrm{u}_{012}^{\prime \prime}+\mathrm{u}_{012}^{\prime}-\mathrm{M}_{1} \mathrm{u}_{012}=-\mathrm{G}_{\mathrm{r}} \theta_{012} \\
\mathrm{u}_{012}^{\prime \prime \prime}+\mathrm{u}_{022}^{\prime \prime}+\mathrm{u}_{022}^{\prime}=-\mathrm{M}_{1} \mathrm{u}_{022} \\
=-\mathrm{G}_{\mathrm{r}} \theta_{022} \\
\theta_{011}^{\prime \prime}+\mathrm{f}_{1} \theta_{011}^{\prime}=0 \\
\theta_{021}^{\prime \prime}+\mathrm{f}_{1} \theta_{021}^{\prime}=0 \\
\theta_{012}^{\prime \prime}+\mathrm{f}_{1} \theta_{012}^{\prime}=\mathrm{f}_{1}\left(\mathrm{u}_{011}^{\prime}\right)^{2} \\
\theta_{022}^{\prime \prime}+\mathrm{f}_{1} \theta_{022}^{\prime}=2 \mathrm{f}_{1} \mathrm{u}_{011}^{\prime} \mathrm{u}_{021}^{\prime}
\end{gathered}
$$

Boundary conditions are

$$
\begin{gathered}
\theta_{011}=\theta_{012}=\theta_{021}=\theta_{022}=0 \\
\mathrm{u}_{011}=\mathrm{u}_{012}=\mathrm{u}_{021}=\mathrm{u}_{022}=0 \text { as } \mathrm{y} \rightarrow 0 \\
\mathrm{u}_{011}=1, \mathrm{u}_{012}=\mathrm{u}_{021}=\mathrm{u}_{022}=0 \\
\theta_{011}=\theta_{012}=\theta_{021}=\theta_{022}=0 \text { as } \mathrm{y} \rightarrow \infty
\end{gathered}
$$

We get the following solutions for the mean temperature profiles as

$$
\begin{array}{r}
\theta_{011}=e^{-f_{1} y} \\
\theta_{012}=G_{r}^{2}\left[b_{2} e^{-f_{1} y}+a_{1}^{2} f_{1} m_{1} e^{-2 m y}+\frac{a_{1}^{2} f_{1} e^{-2 f_{1} y}}{2}\right. \\
\left.-a_{1}^{2} f_{1} f_{2} e^{-\left(m+f_{1}\right) y}\right]
\end{array}
$$

$$
\begin{aligned}
& \theta_{021}=0 \\
& \theta_{022}=2 \mathrm{G}_{\mathrm{r}}^{2} \mathrm{a}_{1} \mathrm{f}_{1}\left[-\left\{\mathrm{m}_{4}+\mathrm{m}_{5}-\mathrm{m}_{6}+\mathrm{f}_{4}\right\} \mathrm{e}^{-\mathrm{f}_{1} \mathrm{y}}\right. \\
& +\left(\mathrm{m}_{5}-\mathrm{m}_{6}\right) \mathrm{e}^{-2 \mathrm{my}} \\
& +\left(m_{4}-f_{2} f_{3}\right) e^{-\left(m+f_{1}\right) y}+\frac{a_{1}^{2} f_{1}^{3} e^{-2 f_{1} y}}{2} \\
& -\mathrm{m}_{0} \mathrm{ye}^{-2 \mathrm{my}} \\
& \left.+\mathrm{m}_{3} \mathrm{ye} \mathrm{e}^{-\left(\mathrm{m}+\mathrm{f}_{1}\right) \mathrm{y}}\right]
\end{aligned}
$$

$$
\begin{aligned}
\theta & =\theta_{0}+\varepsilon \theta_{1} \\
\theta_{0} & =\theta_{01}+\mathrm{R}_{\mathrm{m}} \theta_{02} \\
\theta_{0} & =\left(\theta_{011}+\mathrm{E} \theta_{012}\right)+\mathrm{R}_{\mathrm{m}}\left(\theta_{021}+\mathrm{E} \theta_{022}\right)
\end{aligned}
$$

Mean dimensionless coefficient of heat transfer i.e., Nusselt's number is given by

$\mathrm{Nu}=\left.\theta_{0}^{\prime}\right|_{y=0}$

$=-\mathrm{f}_{1}$

$+E\left[G_{r}^{2}\left\{-b_{2} f_{1}-2 m a_{1}^{2} f_{1} m_{1}-a_{1}^{2} f_{1}^{2}+a_{1}^{2} f_{1} f_{2}\left(m+f_{6}\right)\right\}\right]$

$+R_{m} E\left[\begin{array}{c}2 \mathrm{G}_{\mathrm{r}}^{2} \mathrm{a}_{1} \mathrm{f}_{1}\left\{\mathrm{~m}_{4}+\mathrm{m}_{5}-\mathrm{m}_{6}+\mathrm{f}_{4}\right\} \mathrm{f}_{1} \\ -2 \mathrm{~m}\left(\mathrm{~m}_{5}-\mathrm{m}_{6}\right)- \\ \left(\mathrm{m}+\mathrm{f}_{1}\right)\left(\mathrm{m}_{4}-\mathrm{f}_{2} \mathrm{f}_{3}\right)- \\ \mathrm{a}_{1}^{2} \mathrm{f}_{1}^{4}-\mathrm{m}_{0}+\mathrm{m}_{3}\end{array}\right]$

Now we get the following solutions for the mean velocity profiles.

$$
u_{011}=G_{r} a_{1}\left[e^{-m y}-e^{-f_{1} y}\right]
$$

$\mathrm{u}_{02}$

$=G_{r}\left[f_{1}^{3} a_{1}^{2} e^{-m y}\right.$

$\left.-a_{1} b_{1} y e^{-m y}-a_{1}^{2} f_{1}^{3} e^{-f_{1} y}\right]$ 


$$
\begin{aligned}
\mathrm{u}_{012}=\frac{\mathrm{G}_{\mathrm{r}}^{2}}{\mathrm{~b}_{1}}\left[\left(\mathrm{a}_{8}+\right.\right. & \left.\left.\mathrm{a}_{9}+\mathrm{a}_{10}-\mathrm{a}_{11}\right) \mathrm{e}^{-\mathrm{my}}\right] \\
& -\frac{\mathrm{G}_{\mathrm{r}}^{3}}{\mathrm{~b}_{1}}\left[\left(\mathrm{a}_{8} \mathrm{e}^{-\mathrm{f}_{1} \mathrm{y}}+\mathrm{a}_{9} \mathrm{e}^{-2 \mathrm{f}_{1} \mathrm{y}}\right.\right. \\
& \left.\left.+\mathrm{a}_{10} \mathrm{e}^{-2 \mathrm{my}}-\mathrm{a}_{11} \mathrm{e}^{-\left(\mathrm{m}+\mathrm{f}_{1}\right) \mathrm{y}}\right)\right]
\end{aligned}
$$

$\mathrm{u}_{022}$

$=G_{r}^{3}\left[\left(2 f_{1} a_{7}+a_{16}\right) e^{-m y}+\left(2 f_{1} a_{3}-a_{12}\right) e^{-f_{1} y}\right.$

$-\left(2 \mathrm{f}_{1} \mathrm{a}_{4}+2 \mathrm{f}_{1} \mathrm{a}_{1} \mathrm{~m}_{10}+\mathrm{a}_{14}\right) \mathrm{e}^{-2 \mathrm{my}}-\left(2 \mathrm{f}_{1} \mathrm{a}_{1} \mathrm{a}_{6}\right.$

$\left.+a_{13}\right) e^{-2 f_{1} y}-\left(2 f_{1} a_{1} a_{5}+2 f_{1} a_{1} m_{3} f_{6}-a_{15}\right) e^{-\left(m+f_{1}\right) y}$

$-\left(2 \mathrm{f}_{1} \mathrm{a}_{1} \mathrm{~m}_{9}+\mathrm{a}_{8}+\mathrm{a}_{9}+\mathrm{a}_{10}-\mathrm{a}_{11}\right) \mathrm{ye}^{-\mathrm{my}}$

$\left.-\left(2 \mathrm{f}_{1} \mathrm{a}_{1} \mathrm{~m}_{3} \mathrm{f}_{5}\right) \mathrm{ye}^{-\left(\mathrm{m}+\mathrm{f}_{1}\right) \mathrm{y}}\right]$

$$
\mathrm{u}_{0}=\left(\mathrm{u}_{011}+\mathrm{Eu}_{012}\right)+\mathrm{R}_{\mathrm{m}}\left(\mathrm{u}_{021}+\mathrm{Eu}_{022}\right)
$$

Mean non-dimensional skin friction is given by

$$
\begin{aligned}
& \mathrm{C}_{\mathrm{f}}=\left.\mathrm{u}_{0}^{\prime}\right|_{y=0}=\left[\mathrm{G}_{\mathrm{r}} \mathrm{a}_{1}\left(\mathrm{f}_{1}-\mathrm{m}\right)\right] \\
&+ \\
&+\mathrm{E}\left[\frac{\mathrm{G}_{\mathrm{r}}^{2}}{\mathrm{~b}_{1}}\left\{\mathrm{~m}\left(\mathrm{a}_{11}-\mathrm{a}_{10}-\mathrm{a}_{9}-\mathrm{a}_{8}\right)\right\}\right. \\
&-\frac{\mathrm{G}_{\mathrm{r}}^{3}}{\mathrm{~b}_{1}}\left\{\mathrm{a}_{11}\left(\mathrm{~m}+\mathrm{f}_{1}\right)-2 \mathrm{ma}_{10}-2 \mathrm{a}_{9} \mathrm{f}_{1}\right. \\
&\left.\left.-\mathrm{a}_{8} \mathrm{f}_{1}\right\}\right] \\
&+\mathrm{R}_{\mathrm{m}}\left[\left\{\mathrm{G}_{\mathrm{r}}\left(\mathrm{a}_{1}^{2} \mathrm{f}_{1}^{4}-\mathrm{a}_{1}^{2} \mathrm{f}_{1}^{3} \mathrm{~m}-\mathrm{a}_{1} \mathrm{~b}_{1}\right)\right\}\right. \\
&+E \mathrm{G}_{\mathrm{r}}^{3}\left\{-\mathrm{m}\left(2 \mathrm{f}_{1} \mathrm{a}_{7}+\mathrm{a}_{16}\right)\right. \\
&-\mathrm{f}_{1}\left(2 \mathrm{f}_{1} \mathrm{a}_{3}-\mathrm{a}_{12}\right) \\
&+2 \mathrm{~m}\left(2 \mathrm{f}_{1} \mathrm{a}_{4}+2 \mathrm{f}_{1} \mathrm{a}_{1} \mathrm{~m}_{10}+\mathrm{a}_{14}\right) \\
&+2 \mathrm{f}_{1}\left(2 \mathrm{f}_{1} \mathrm{a}_{1} \mathrm{a}_{6}+\mathrm{a}_{13}\right) \\
&+\left(\mathrm{m}+\mathrm{f}_{1}\right)\left(2 \mathrm{f}_{1} \mathrm{a}_{1} \mathrm{a}_{5}+2 \mathrm{f}_{1} \mathrm{a}_{1} \mathrm{~m}_{3} \mathrm{f}_{6}\right. \\
&\left.-\mathrm{a}_{15}\right) \\
&-\left(2 \mathrm{f}_{1} \mathrm{a}_{1} \mathrm{~m}_{9}+\mathrm{a}_{8}+\mathrm{a}_{9}+\mathrm{a}_{10}-\mathrm{a}_{11}\right) \\
&\left.\left.-2 \mathrm{f}_{1} \mathrm{a}_{1} \mathrm{~m}_{3} \mathrm{f}_{5}\right\}\right]
\end{aligned}
$$

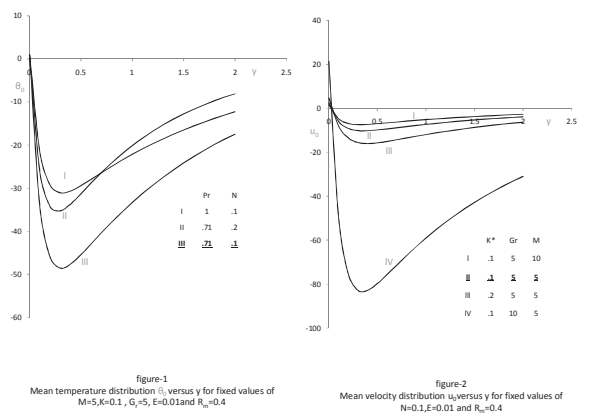

NUSSELT'S NUMBER TABLE 1

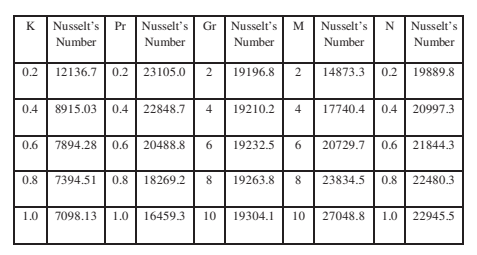

For fixed values of $\mathrm{K}=0.1, \mathrm{Pr}=0.71, \mathrm{Gr}=5, \mathrm{M}=5, \mathrm{~N}=0$. land

SKIN FRICTION TABLE 2

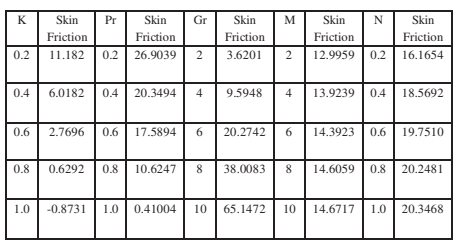

For fixed values of $\mathrm{K}=0.1, \mathrm{Pr}=0.71, \mathrm{Gr}=5, \mathrm{M}=5, \mathrm{~N}=0$. land
\[ \mathrm{Rm}=0.4 \]

\section{CONCLUSIONS}

We observe that as permeability parameter $\mathrm{K}$ and Groshoff number $G_{\mathrm{r}}$ suppress velocity distribution it is due to the fact that in Rosseland's approximation the temperature differences are very small, that is why adverse trend is noted. In general permeability parameter $\mathrm{K}$ and Groshoff number $\mathrm{G}_{\mathrm{r}}$ influence velocity distribution, whereas Lorentz force influences the velocity distribution because of the Roseland's approximation refer fig-2

We see that temperature is in direct proportion to radiation parameter $\mathrm{N}$ from (2.8), so the effect of radiation parameter $\mathrm{N}$ is to influence the temperature distribution and also we note that as Prandle number $\mathrm{P}_{\mathrm{r}}$ increases temperature distribution increases refer fig-1

The effect of $\mathrm{K}$ (Permeability parameter) and $\mathrm{P}_{\mathrm{r}}$ (Prandle number) is to decrease the heat transfer whereas $\mathrm{G}_{\mathrm{r}}$ (Grashoff number), M (Hartmann number) and $\mathrm{N}$ (Radiation parameter) is to influence the heat transfer. The above parameters have the same effect on skin friction refer table $1 \& 2$. 


\section{REFERENCES}

[1] M.J. Lighthill "The response of laminar skin friction and heat transfer to fluctuation in the stream velocity" Proceedings of the Royal Society, A 224,1. (1954)

[2] M.Q. Brewester, "Thermal radiative transfer and properties" New York, John Wiley and Sons Inc. (1992)

[3] M.A. Seddeek, "Effects of radiation and variable viscosity on a MHD free convection flow past a semi-infinite flat plate with an aligned magnetic field in the case of unsteady flow" International Journal of Heat and Mass transfer 45 (2002) 931-935

[4] M.K. Mazumdar and R.K. Deka, "MHD flow past an impulsively started infinite vertical plate in presence of thermal radiation", Romanian Journal of Physics, 52(57), (2007) 565-573

[5] I.U. Mbeledogu and A. Ogulu, "Heat and mass transfer of an unsteady MHD natural convection flow of a rotating fluid past a vertical porous flat plate in the presence of radiative heat transfer" International Journal of Heat and Mass Transfer 50 (2007) 1902-1908

[6] I.U. Mbeledogu, A.R.C. Amakiri and A. Ogulu, "Unsteady MHD free convective flow of a compressible fluid past a moving vertical plate in the presence of radiative heat transfer" International Journal of Heat and Mass Transfer 50 (2007) 1668-1674

[7] R.A. Mohamed and S.M. Abo-Dahab, "Influence of chemical reaction and thermal radiation on the heat and mass transfer in MHD micro polar flow over a vertical moving porous plate in a porous medium with heat generation" International Journal of Thermal Sciences 48 (2009) 1800-1813

[8] C. Y. Han, "Hydromagnetic free convection of a radiating fluid" International Journal of Heat and Mass Transfer 52 (2009) 5895-5908

[9] J. Zueco and O. A. Beg "Network simulation solutions for laminar radiating dissipative magneto-gas dynamic heat transfer over a wedge in non-Darcian porous regime" Mathematical and Computer Modelling 50 (2009) 439 452

[10] N. Ahmed and H.K. Sarmah, "Thermal radiation effect on a transient MHD flow with mass transfer past an impulsively fixed infinite vertical plate", International Journal of Applied Mathematics and Mechanics 5 (2009) 8798
[11] A. Ishak, "Mixed convection boundary layer flow over a horizontal plate with thermal radiation" Heat Mass Transfer 46 (2009) 147-151.

[12] Bo-C. Tai and M.-I. Char, "Soret and Dufour effects on free convection flow of non- Newtonian fluids along a vertical plate embedded in a porous medium with thermal radiation" International Communications in Heat Mass Transfer 37 (2010) 480-483.

[13] C.I.Cookey and C. Nwaigwe, "Unsteady MHD flow of a radiating fluid over a moving heated porous plate with time - dependent suction", American Journal of Scientific and Industrial Research, (2010) 1(1): 88 95.

[14] M. Narahari, "Effects of thermal radiation and free convection currents on the unsteady Couette flow between two vertical parallel plates with constant heat flux at one boundary", World Scientific and Engineering Academy and Society Transactions on Heat and Mass Transfer, (2010) 1(5): 21 - 30.

[15] C.I Cookey, V. B. O. Pepple and I. Tamunobereton-ari "On steady hydromagnetic flow of a radiating viscous fluid through a horizontal channel in a porous medium" American Journal of Scientific and Industrial Research, (2010), 1(2): 303-308

[16] N.P. Singh, A.K. Singh, A.K. Singh and P. Agnihotri, "Effects of thermophoresis on hydromagnetic mixed convection and mass transfer flow past a vertical permeable plate with variable suction and thermal radiation" Communications in Nonlinear Science and Numerical Simulation 16 (2011) 2519-2534

[17] N. Ahmed, "Soret and radiation effects on transient MHD free convection from an impulsively started infinite vertical plate" J. Heat Transfer 134 (2012) 062701-1-062701-9.

[18] M. Turkyilmazoglu and I. Pop, "Soret and heat source effects on the unsteady radiative MHD free convection flow from an impulsively started infinite vertical plate" International Journal of Heat and Mass Transfer 55 (2012) 7635-7644

[19] M. Ferdows, M.J. Uddin and A.A. Afify, "Scaling group transformation for MHD boundary layer free convective heat and mass transfer flow past a convectively heated nonlinear radiating stretching sheet" International Journal of Heat and Mass Transfer 56 (2013)

$181-187$ 